\title{
Myocarditis due to systemic lupus erythematosus associated with cardiogenic shock
}

Sohaib Tariq, Anjali Garg, Alan Gass, Wilbert S. Aronow

Cardiology Division, Department of Medicine, Westchester Medical Center/New York Medical College, Valhalla, New York, USA

Submitted: 14 October 2015

Accepted: 4 November 2015

Arch Med Sci 2018; 14, 2: 460-462

DOI: https://doi.org/10.5114/aoms.2017.68692

Copyright $\odot 2017$ Termedia \& Banach

Systemic lupus erythematosus (SLE) is a systemic autoimmune disease, known to involve different organs including the heart. One of the common cardiac manifestations is pericarditis [1]. Other forms of cardiac involvement include coronary artery disease, arteritis and valvular disease. One of the rare presentations of systemic lupus erythematosus include myocarditis and once suspected, should be diagnosed and treated promptly to avoid fatal consequences [2]. We report an interesting case of a patient with a history of SLE presenting with cardiogenic shock from acute myocarditis, requiring advanced heart failure therapies.

A 41-year-old Caucasian male with a history of SLE diagnosed 2 years ago on treatment with hydroxychloroquine, presented to an outside hospital with complains of worsening shortness of breath and fatigue for 1 week. On initial assessment, significant findings on examination were mild confusion, jugular venous distention, crepitant rales at bases of both the lungs, a grade 3/6 holo-systolic murmur heard at the apex which radiated to the left axilla and cool extremities. His heart rate was 112 beats per minute, blood pressure $98 / 62 \mathrm{~mm} \mathrm{Hg}$, respiratory rate 29 breaths per minute, serum creatinine $1.7 \mathrm{mg} / \mathrm{dl}$, aspartate transaminase (AST) $4539 \mathrm{U} / \mathrm{l}$, alanine transaminase (ALT) $4264 \mathrm{U} / \mathrm{l}$, serum total bilirubin $1.5 \mathrm{mg} / \mathrm{dl}$, international normalized ratio (INR) 2.8 and serum lactate $5.4 \mathrm{mmol} / \mathrm{l}$. Inotropic support with dobutamine was started. Two-dimensional echocardiography revealed severe biventricular failure with global hypokinesis and left ventricular ejection fraction of $15 \%$. Left ventricle was dilated $(6.3 \mathrm{~cm})$ and an apical thrombus was present. Left heart catheterization demonstrated non-obstructive coronary artery disease. Right heart catheterization (RHC) on inotropic support showed a low output state, and an intra-aortic balloon pump (IABP) was placed for mechanical circulatory support. He continued to be in refractory cardiogenic shock and was transferred to Westchester Medical Center for further therapy. On arrival to our facility, the patient was hemodynamically unstable requiring multiple vasopressor agents and IABP. He continued to have evidence of end organ malperfusion. The decision was made to escalate mechanical circulatory support to venous-arterial extracorporeal membrane oxygenation (V-A ECMO) as a bridge to recovery or decision. Due to a high clinical suspicion for lupus myocarditis, treatment with steroids, IVIG and Rituximab, was initiated. Right heart catheterization on ECMO support showed a central venous pressure of $9 \mathrm{~mm} \mathrm{Hg}$, a pulmonary artery (PA) pressure of 27/16 mm Hg (mean: $20 \mathrm{~mm} \mathrm{Hg}$ ), a pulmonary capillary wedge pressure of 12, a PA sat-

\author{
Corresponding author: \\ Wilbert S. Aronow MD, FACC, \\ FAHA \\ Cardiology Division \\ New York Medical College \\ Macy Pavilion \\ Room 148 \\ Valhalla, NY 10595, USA \\ Phone: (914) 493-5311 \\ Fax: (914) 235-6274 \\ E-mail:wsaronow@aol.com
}


uration of $68 \%$, cardiac output (Fick) of $8.5 \mathrm{l} / \mathrm{min}$ and a cardiac index of $3.8 \mathrm{l} / \mathrm{min} / \mathrm{m}^{2}$.

Endomyocardial biopsy showed no evidence of significant interstitial mononuclear cell infiltration, intracellular depositions or myocardial necrosis. Trichome stain for amyloidosis was negative. The serum ferritin level was normal, and the human immunodeficiency virus antibody was negative. A complete autoimmune work-up was pursued including anti-double-stranded DNA antibody, anti-cardiolipin antibody, anti-Sjögren's-syndrome-related antigen antibody, anti-Smith, anti-ribonucleoprotein antibody and lupus anticoagulant, which were all undetectable. Serum antinuclear antibody (ANA) was positive with a speckled pattern consistent with systemic lupus erythematosus.

Repeat assessment of the left ventricle function by two-dimensional echocardiography showed little improvement. End organ function including liver enzymes and creatinine also improved. Attempts were made to wean off the ECMO support which were unsuccessful. A heart mate II was implanted as a bridge to heart transplantation along with tricuspid valve repair. No complications were seen while the patient was on mechanical circulatory support. He survived to hospital discharge, and two years later underwent an orthotopic heart transplant without any post-operative complications.

Myocardial dysfunction in SLE is usually multifactorial and may result from immunological injury, ischemia, valvular disease, or coexisting problems such as hypertension. Although uncommon, myocarditis is a serious manifestation of SLE, with a clinical prevalence of about $9 \%$. However, it has been shown to be present in $57 \%$ of post mortem analyses, suggesting a high prevalence of subclinical disease [3]. Myocarditis has a varied clinical manifestation and can present with dyspnea, non-exertional chest pain, peripheral edema, fever, orthopnea, diaphoresis, paroxysmal nocturnal dyspnea, nausea, vomiting, or palpitations [4].

The gold standard for confirmation of myocarditis is endomyocardial biopsy. However, the focal nature of the disease in lupus myocarditis makes it less sensitive. The biopsy generally reveals patches of myocardial fibrosis, sparse interstitial mononuclear cell infiltrates, and occasional myocyte necrosis with immune complex deposition, even in areas devoid of inflammatory changes. The diagnosis is often achieved clinically and with the use of diagnostic imaging. Echocardiography and cardiac magnetic resonance imaging (MRI) are commonly used to assess biventricular function [5]. Cardiac MRI can also detect the presence of necrosis or scar in myocarditis by late gadolinium enhancement. Left ventricular dysfunction is usually global, but can present as regional wall motion abnormalities, chamber dilation or diastolic dysfunction [6, 7]. Part of the difficulty arising in diagnosis of lupus myocarditis is that none of the current diagnostic modalities have established sensitivity or specificity. Management aspects of the disease are also not well studied.

Myocarditis is a rare but fatal complication of SLE. The relative rarity of the condition makes it difficult to conduct controlled trials and various managements have been described in isolated case reports. High-dose corticosteroid treatment is the most common therapy used for lupus myocarditis [8, 9]. It appears to be an effective initial treatment resulting in improvement of left ventricular function in hemodynamically stable patient but there are very few cases of lupus myocarditis presenting as cardiogenic shock requiring mechanical support. Other drugs used in treatment are azathioprine, cyclophosphamide, rituximab and intravenous immunoglobulins. Our patient was treated with intravenous immunoglobulins, rituximab and glucocorticoids, which resulted in little improvement of the left ventricle function. After undergoing orthotopic heart transplantation, our patient showed no evidence of recurrence of lupus myocarditis on subsequent endomyocardial biopsies and imaging during a follow-up of 24 months. The risk of recurrence of lupus myocarditis in the transplanted heart has not been established.

In conclusion, lupus myocarditis can present as a life threatening complication. Its diagnosis is primarily based on clinical suspicion as diagnostic tools are not sensitive. Treatment is still not well established. We describe a case of lupus myocarditis complicated by cardiogenic shock requiring mechanical circulatory support.

\section{Conflict of interest}

The authors declare no conflict of interest.

\section{References}

1. Moder KG, Miller TD, Tazelaar HD. Cardiac involvement in systemic lupus erythematosus. Mayo Clin Proc 1999; 74: 275-84.

2. Apte M, Mcgwin G, Vilá LM, et al. Associated factors and impact of myocarditis in patients with SLE from LUMINA, a multiethnic US cohort (LV). [corrected]. Rheumatology (Oxford) 2008; 47: 362-7.

3. Wijetunga M, Rockson S. Myocarditis in systemic lupus erythematosus. Am J Med 2002; 113: 419-23.

4. Cooper LT. Myocarditis. N Engl J Med 2009; 360: 1526-38.

5. Mahrholdt H, Goedecke C, Wagner A, et al. Cardiovascular magnetic resonance assessment of human myocarditis: a comparison to histology and molecular pathology. Circulation 2004; 109: 1250-8.

6. Enomoto K, Kaji Y, Mayumi T, et al. Frequency of valvular regurgitation by color Doppler echocardiography in 
systemic lupus erythematosus. Am J Cardiol 1991; 67: 209-11.

7. Sasson Z, Rasooly Y, Chow CW, Marshall S, Urowitz MB. Impairment of left ventricular diastolic function in systemic lupus erythematosus. Am J Cardiol 1992; 69: 1629-34.

8. Zhang L, Zhu YL, Li MT, et al. Lupus myocarditis: a casecontrol study from China. Chin Med J 2015; 128: 2588-94.

9. Barnado A, Kamen DL. Myocarditis successfully treated with intravenous immunoglobulin in a patient with systemic lupus erythematous and myositis. Am J Med Sci 2014; 347: 256-7. 